\title{
Whole-cell matrix-assisted laser desorption/ionization time-of- flight mass spectrometry for rapid identification of bacteria cultured in liquid media
}

\author{
ZHOU Na ${ }^{1 \dagger}$, WANG Na ${ }^{2 \dagger}$, XU Bin ${ }^{2}$, WANG Jie ${ }^{2}$, FANG JunJian ${ }^{2}$, DONG FangTing ${ }^{2}$, \\ $\mathrm{HE} \mathrm{Kun}^{2 *} \&$ YANG XiaoHong ${ }^{1 *}$ \\ ${ }^{1}$ Pharmaceutical Department of Jilin University, Changchun 130021, China; \\ ${ }^{2}$ National Center of Biomedical Analysis, Beijing 100850, China
}

Received May 26, 2010; accepted June 13, 2010

\begin{abstract}
Matrix-assisted laser desorption/ionization time-of-flight mass spectrometry (MALDI-TOF MS) has been used for many years to rapidly identify whole bacteria. However, no consistent methodology exists for the rapid identification of bacteria cultured in liquid media. Thus, in this study we explored the use of MALDI-TOF MS analysis for rapid identification of cells cultured in liquid media. We determined that 2,5-dihydroxybenzoic acid $\left(50 \mathrm{mg} \mathrm{mL}^{-1}, 50 \%\right.$ acetonitrile, $0.1 \%$ trifluoroacetic acid) was the best matrix solution for MALDI-TOF MS for this type of study. Moreover, the tested strains were successfully differentiated by principal component analysis, and the main characteristics of the mass peaks for each species were found in mixed culture samples. In addition, we found that the minimum number of cells for detection was $1.8 \times 10^{3}$. In conclusion, our findings suggest that MS-based techniques can be developed as an auxiliary method for rapidly and accurately identifying bacteria cultured in liquid media.
\end{abstract}

MALDI-TOF MS, bacteria, PCA, identification

Citation: Zhou N, Wang N, Xu B, et al. Whole-cell matrix-assisted laser desorption/ionization time-of-flight mass spectrometry for rapid identification of bacteria cultured in liquid media. Sci China Life Sci, 2011, 54: 48-53, doi: 10.1007/s11427-010-4119-9

Efficient methods for classifying and identifying bacteria are important for many applications in microbiology, and especially for emergency bio-safety analysis in which the rapid and accurate identification of microorganisms is necessary [1]. Many effective methods have previously been developed for bacterial identification. For instance, a variety of traditional microbiologic methods, such as microbiologic procedures, antibody recognition, and polymerase chain reaction amplification, provide high sensitivity and specificity, but these methods are time-consuming, typically requiring more than $48 \mathrm{~h}$ to complete [2,3]. In 1975, Anhalt and Fenselau [4] proposed the application of matrix-assisted

$\dagger$ Contributed equally to this work

*Corresponding author (email: hk_ncba@126.com; xiaohongyang88@126.com) laser desorption/ionization time-of-flight mass spectrometry (MALDI-TOF MS) for characterization and identification of bacterial species. Given its shorter analysis time, low sample requirement, high resolution, sensitivity, and reproducibility, it has been extensively used as a rapid, inexpensive, and accurate method for bacterial identification [5-9]. Different research groups have adopted different MALDITOF MS approaches, such as inclusion of various sample pretreatments and matrices [10-12], although most of these methods use bacteria cultured on an agar plate until they grow into pure colonies. Unfortunately, the monoclonal bacterial culture necessary for such approaches requires almost $24 \mathrm{~h}$, and a systematic method for mass spectrometric analysis of bacteria cultured in liquid media, which takes just a few hours, has yet to be established. 
In the present study, bacterial strains were first grown in liquid media for $8 \mathrm{~h}$ and were inactivated by $75 \%$ ethanol. An orthogonal experiment was then used to develop an efficient matrix for MALDI-TOF MS analysis. Eleven standard bacteria strains belonging to three different species were subsequently used to study classification efficiency.

\section{Materials and methods}

\subsection{Instrument and chemicals}

\subsubsection{Instrument}

We used an Ultraflex TOF/TOF mass spectrometer, 384 MTP target plates (Bruker Daltonics, Bremen, Germany), a GHP-9270 incubator (Blue Pard, Shanghai, China), a MiniSpin Plus centrifuge (Eppendorf, Germany), and a ZHWY-200B rocking incubator (Zhi Cheng, Shanghai, China).

\subsubsection{Chemicals}

$\alpha$-cyano-4-hydroxycinnamic acid (CHCA), 2,5-dihydroxybenzoic acid (DHB), sinapic acid (SA), and standard peptides were purchased from Bruker Daltonics. Insulin and cytochrome $\mathrm{C}$ were purchased from Sigma Chemical Co. (St. Louis, MO, USA). Acetonitrile (ACN) was purchased from J. T. Baker (Phillipsburg, NJ, USA). Trifluoroacetic acid (TFA) was purchased from Acros Organics (Geel, Belgium). Agar, tryptone, and yeast extract were purchased from Oxoid (Basingstoke, UK). $\mathrm{NaCl}$ and $\mathrm{NaOH}$ (analytically pure) were purchased from Beijing Chemical Works (Beijing, China).

\subsection{Bacterial strains}

The strains used in this study were provided by the State Key Laboratory of Pathogen and Biosecurity of China and are listed in Table 1. The laboratory strain of Escherichia

Table 1 Bacterial strains

\begin{tabular}{ccc}
\hline Organism & Strain & Source \\
\hline & JM109 & Lab collection \\
Escherichia coli & 8739 & CMCC $^{\text {a }}$ \\
& 44708 & CMCC \\
Shigella boydii & 44737 & CMCC \\
Shigella sonnei & 25922 & CMCC \\
Shigella flexneri & 51265 & CMCC \\
& 51344 & CMCC \\
Salmonella paratyphi & 51062 & CMCC \\
& 50671 & CMCC \\
& 50094 & CMCC \\
& 50677 & CMCC \\
& 50095 & CMCC \\
\hline
\end{tabular}

a) CMCC, National Center for Medical Culture Collections. coli JM109 was used for optimization of experimental conditions.

\subsection{Matrix solutions}

Table 2 shows the schedule that we used for the orthogonal test of the matrix solutions. Different matrices (A), matrix concentrations (B), and matrix solvents (C) were selected as the three factors for analysis.

\subsection{Sample preparation}

Each bacterial strain was first inoculated at $1 \%$ into a sample tube containing $5 \mathrm{~mL}$ Luria-Bertani medium (10 g tryptone, $5 \mathrm{~g}$ yeast extract, $10 \mathrm{~g} \mathrm{NaCl}$, and $100 \mu \mathrm{L} 10 \mathrm{mmol} \mathrm{L}^{-1}$ $\mathrm{NaOH}$ in $1 \mathrm{~L}$ of water) and was incubated with shaking for $8 \mathrm{~h}$ at $37^{\circ} \mathrm{C}$. Subsequently, $1 \mathrm{~mL}$ of each sample in a $1.5 \mathrm{~mL}$ tube was centrifuged at $6000 \times g$ at room temperature for 3 min and the supernatant was then discarded. To inactivate the bacteria, we re-suspended each bacterial pellet in 250 $\mu \mathrm{L}$ of water mixed with $750 \mu \mathrm{L}$ of ethanol, centrifuged each tube at $6000 \times g$, and discarded the supernatant. We then washed each pellet with $1 \mathrm{~mL}$ of water, centrifuged it at $6000 \times g$, and discarded the supernatant. Moreover, we repeated this last step one additional time. Finally, we re-suspended each strain of cells with $1 \mathrm{~mL}$ of water. A $1-\mu \mathrm{L}$ sample of bacteria was then mixed with $1 \mu \mathrm{L}$ of matrix solution and was loaded onto the target plate, which was subsequently allowed to dry in air at ambient temperature [13]. Three spots were prepared for each sample.

\subsection{Mass spectrometry}

Positive-ion mass spectra were obtained on an Ultraflex TOF/TOF mass spectrometer equipped with a $\mathrm{N}_{2} 337 \mathrm{~nm}$ laser for each sample. The mass spectrometer was operated in linear mode at a $22 \mathrm{kV}$ accelerating voltage. Each mass spectrum was the sum of the ions obtained from 2000 laser shots performed in 10 different regions of the same well at a laser intensity of $40 \%$ (and are presented in arbitrary units $(A U))$. The spectra were analyzed in a mass range of 2000-20000 Da. The mass spectra were calibrated using peptides and protein mixtures containing 1 pmol of each of

Table 2 Factors and levels selected for the orthogonal experiment ${ }^{\text {a) }}$

\begin{tabular}{|c|c|c|c|}
\hline Level & A: matrix & $\begin{array}{c}\text { B: matrix concentration } \\
\left(\mathrm{mg} \mathrm{mL}^{-1}\right)\end{array}$ & C: matrix solvent \\
\hline 1 & $\mathrm{CHCA}$ & 5 & $\begin{array}{c}0.1 \% \text { TFA; } 50 \% \text { acetoni- } \\
\text { trile; } 49.9 \% \text { water }\end{array}$ \\
\hline 2 & DHB & 10 & $\begin{array}{c}0.1 \% \text { TFA; } 40 \% \text { acetoni- } \\
\text { trile; } 59.9 \% \text { water }\end{array}$ \\
\hline 3 & SA & 50 & $\begin{array}{c}0.1 \% \text { TFA; } 30 \% \text { acetoni- } \\
\text { trile; } 69.9 \% \text { water }\end{array}$ \\
\hline
\end{tabular}

a) CHCA, $\alpha$-cyano-4-hydroxycinnamic acid; DHB, 2,5-dihydroxybenzoic acid; SA, sinapic acid; TFA, trifluoroacetic acid. 
the standard peptides $(1047.19,1297.48,1348.64,1620.86$, 1760.02, 2094.43, 2466.68, and $3149.57 \mathrm{Da})$, insulin (5733.5 Da), and cytochrome C (12360.5 Da) to achieve a mass accuracy of $0.1 \%$.

\subsection{Estimation of the viable cell number}

The bacterial samples were serially diluted to ensure precise counting of individual cells, and the viable cell numbers were determined as colony forming units (CFUs) by the drop plate method [14].

\subsection{Data analysis}

All qualified mass peaks (signal-to-noise ratio $>5$ ) were compiled by FlexAnalysis 3.0 software (Bruker Daltonics). ClinProTool $^{\mathrm{TM}} 2.1$ software (Bruker Daltonics) was used for principal component analysis (PCA).

\section{Results}

\subsection{Selection of the matrix solution for MALDI-TOF MS analysis}

To determine the most effective matrix for sample preparation, we used an orthogonal experiment to optimize the different matrices. A detailed scheme of the orthogonal test is shown in Table 3 . The criteria we used in the evaluation were the number of peaks observed, signal strength, resolution, and reproducibility; however, only the number of peaks was taken as the index point for evaluation of the matrix performance under different factors and levels. The type of matrix factor showed the most notable influence on the mass spectra. Moreover, all of these mass spectra showed high resolution and reproducibility, but the mass spectra of DHB matrices had the best signal strength. The most effective matrix was series No. $6\left(50 \mathrm{mg} \mathrm{mL}^{-1} \mathrm{DHB}\right.$ in $50 \%$ acetonitrile with $0.1 \%$ TFA). The mass spectra of the bacterial suspensions generally exhibited a mass-to-charge ratio $(\mathrm{m} / \mathrm{z})$ from 2000 to 16000 , with high spectra reproducibility, little noise, and a flat baseline. A $1-\mu \mathrm{L}$ medium sample was also analyzed to exclude interference of the culture medium from analysis (Figure 1).

Table 3 Detailed scheme of $\mathrm{L}_{9}\left(3^{3}\right)$ orthogonal test and the mean number of peaks of the three spots for one sample

\begin{tabular}{ccccc}
\hline $\begin{array}{c}\text { Series } \\
\text { No. }\end{array}$ & $\begin{array}{c}\text { Level of } \\
\text { A }\end{array}$ & $\begin{array}{c}\text { Level of } \\
\text { B }\end{array}$ & $\begin{array}{c}\text { Level of } \\
\text { C }\end{array}$ & $\begin{array}{c}\text { Mean number of } \\
\text { peaks }(n=3)\end{array}$ \\
\hline 1 & 1 & 1 & 1 & 81 \\
2 & 1 & 2 & 2 & 77 \\
3 & 1 & 3 & 3 & 70 \\
4 & 2 & 1 & 2 & 122 \\
5 & 2 & 2 & 3 & 115 \\
6 & 2 & 3 & 1 & 131 \\
7 & 3 & 1 & 3 & 88 \\
8 & 3 & 2 & 1 & 107 \\
9 & 3 & 3 & 2 & 91 \\
\hline
\end{tabular}

\subsection{Reproducibility of mass spectra analyzed by MALDI-TOF MS}

To evaluate the reproducibility of the MALDI-TOF MS method used in this study, E. coli JM109 was cultured in three different sample tubes collected at different times in the same day. The samples were mixed with the optimized matrix solution $\left(50 \mathrm{mg} \mathrm{mL}^{-1} \mathrm{DHB}\right.$ in $50 \%$ acetonitrile with $0.1 \%$ TFA) and were analyzed by MALDI-TOF MS independently. The relative peak intensities of six peaks representing low-, medium-, and high-abundance peptides over the whole mass range were determined. The coefficient of variation of the six selected peaks was $8.65 \%-18.92 \%$ (Table 4).

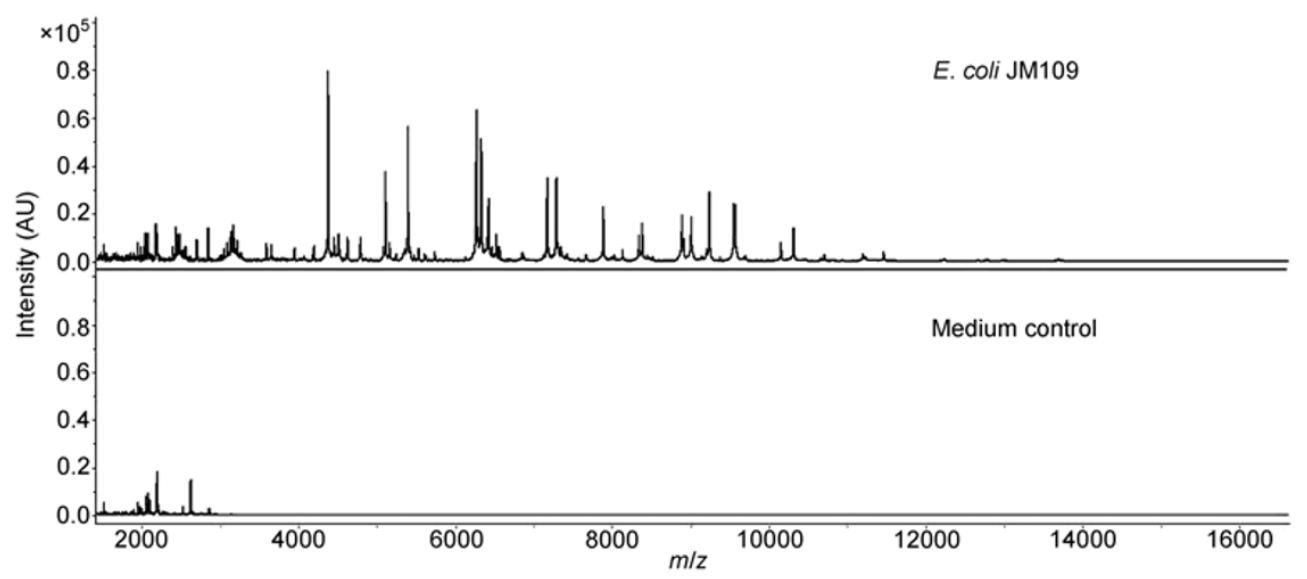

Figure 1 Mass spectra of Escherichia coli JM109 and the medium mixed with matrix series No. 6 analyzed by matrix-assisted laser desorption/ionization time-of-flight mass spectrometry. The intensities (in arbitrary units (AU)) of the ions are shown on the $y$-axis, and the mass-to-charge ratios ( $m / z$ ) are shown on the $x$-axis. 
Table 4 Reproducibility of mass spectra analyzed by matrix-assisted laser desorption/ionization time-of-flight mass spectrometry

\begin{tabular}{ccc}
\hline$m / z^{\mathrm{a})}$ & $\mathrm{MRI}^{\mathrm{b})}(\%)$ & $\mathrm{CV}^{\mathrm{c})}(\%)$ \\
\hline 2686.05 & 4.67 & 12.37 \\
3635.16 & 3.33 & 17.32 \\
5098.45 & 19.67 & 12.80 \\
6259.52 & 33.00 & 18.92 \\
7164.27 & 17.67 & 8.65 \\
8376.70 & 8.33 & 13.86 \\
\hline
\end{tabular}

a) Mass-to-charge ratio $(\mathrm{m} / \mathrm{z})$ values are average molecular weights. b) MRI, mean relative intensity. c) $\mathrm{CV}$, coefficient of variation.

\subsection{Recognition of bacteria analyzed by MALDI-TOF MS}

Consistent with previous research, we performed PCA on the mass spectral data to differentiate the bacteria studied $[15,16]$. Eleven standard strains belonging to three different species were analyzed to evaluate the recognition of the
MALDI-TOF MS analysis. We spotted each bacterial sample on three different positions of the MALDI target, and all mass spectral data were analyzed together. We determined that the strains were separated by species (Figure 2A). There was small separation between E. coli and Shigella spp. compared with the separation of both from Salmonella paratyphi. The peaks shown in Figure 2B were the main characteristic peaks of each species. For $E$. coli, unique signals were observed near $\mathrm{m} / \mathrm{z} 5095$ and 9746. For the Shigella spp., signals were observed near $\mathrm{m} / \mathrm{z}$ 5076. However, for $S$. paratyphi, signals were observed near $\mathrm{m} / \mathrm{z}$ 5611, 6067, and 6092. All of these strains were also cultured in a single sample tube and the mixed cell suspension was analyzed. In the mixed sample, the main characteristic peaks of each of the species were found (Figure 2C). These results strongly suggest the potential of identifying different bacterial species in complex samples without the requirement of additional operations.

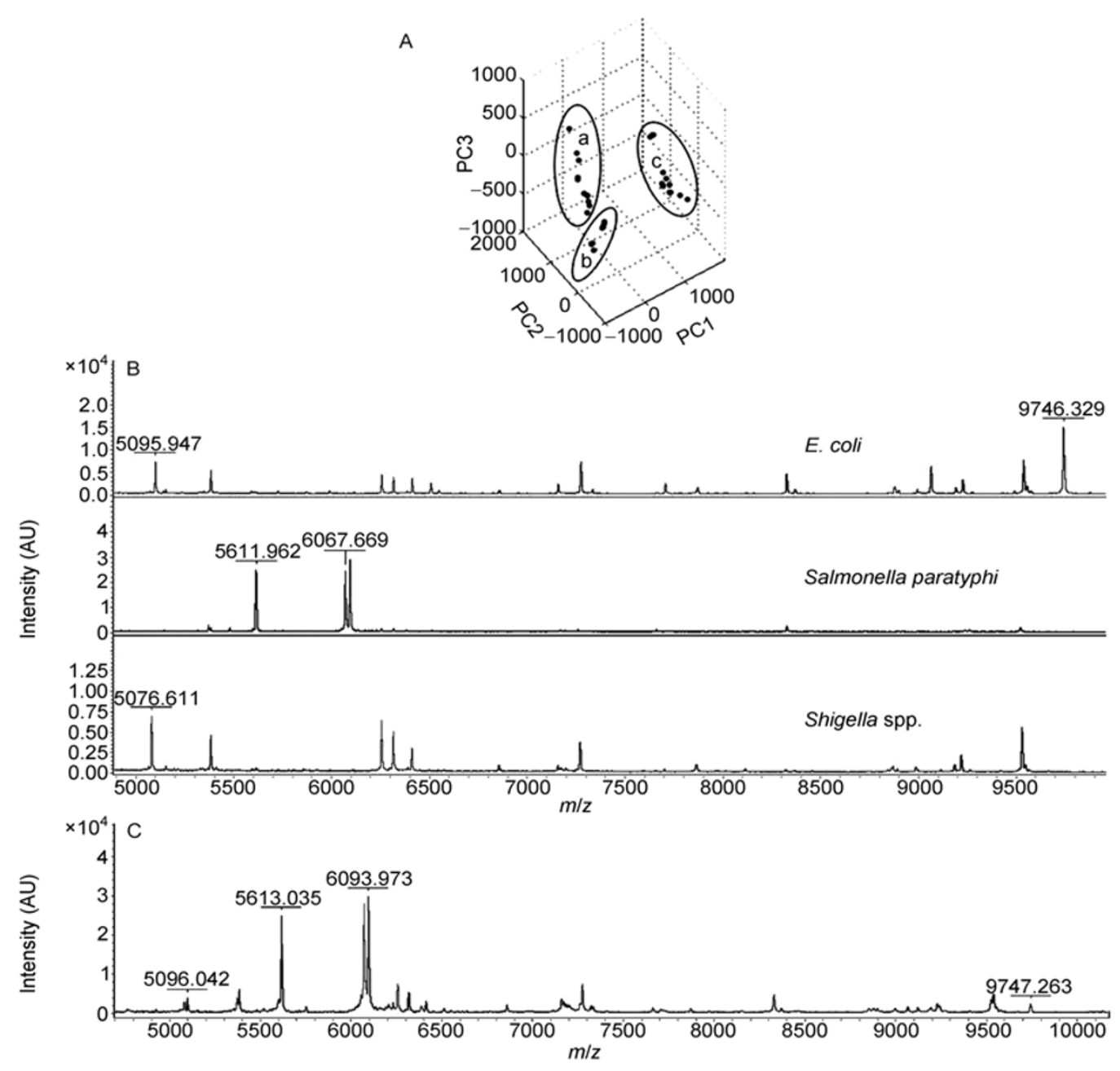

Figure 2 Principal component analysis (PCA) plots (A) and the main characteristic mass peaks of the analyzed strains (B and C). A, PCA spots of all of the 33 mass spectra are shown. The spots in cycles a, b, and c represent the strains of Escherichia. coli, Shigella spp., and Salmonella paratyphi, respectively. B, Unique signals for E. coli, Shigella spp., and S. paratyphi. C, The main characteristic mass peaks of each species detected in their mixed sample. The intensities (in arbitrary units (AU)) of the ions are shown on the $y$-axis, and the mass-to-charge ratios $(\mathrm{m} / \mathrm{z}$ ) are shown on the $x$-axis. 


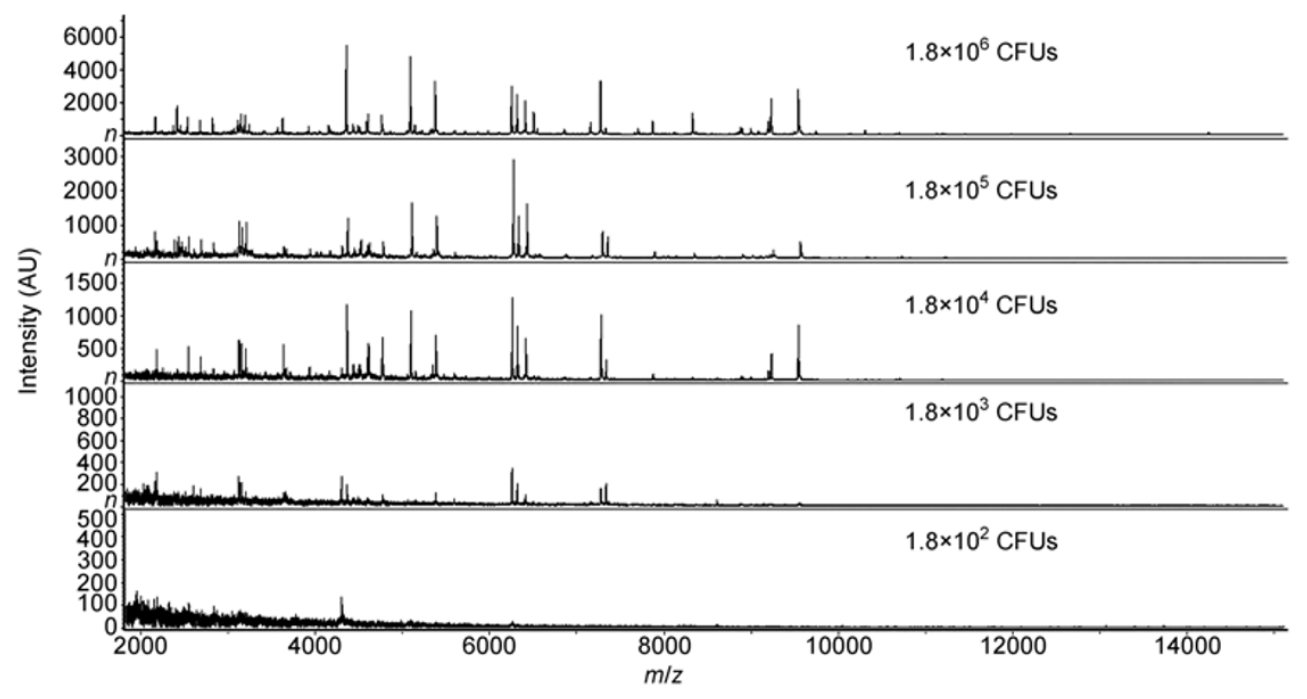

Figure 3 Matrix-assisted laser desorption/ionization time-of-flight mass spectrometry analysis of series dilutions of Escherichia coli JM109 to determine detection sensitivity. The intensities (in arbitrary units (AU)) of the ions are shown on the $y$-axis, and the mass-to-charge ratios ( $m / z$ ) are shown on the $x$-axis.

\subsection{Detection sensitivity of MALDI-TOF MS analysis of serial dilutions of $E$. coli JM109}

To determine the sensitivity of our approach for bacterial identification, we used a $1 \mu \mathrm{L}$ preparation of E. coli JM109 for MS profiling, which contained approximately $1.8 \times 10^{6}$ cells. Through serial dilution, we found that $1.8 \times 10^{3}$ was the minimum number of cells for bacterial detection using this technique (Figure 3). We also assessed cells cultured for different periods of time in this study, and determined that cells cultured for $\sim 3 \mathrm{~h}$ could be detected (data not shown).

\section{Discussion}

The identification of pathogenic microorganisms is particularly important for the diagnosis of bacterial infections and for the recognition of various biological and environmental hazards. Samples collected from foodstuffs and drugs almost always contain mixed flora. For the traditional microbiologic assessment methods, cells must be separated and cultured until pure colonies are obtained. In this study, we examined different bacterial species (alone or in combination) by MALDI-TOF MS, and found that the main characteristic mass peaks of the individual species could be accurately identified in mixed flora. Our findings strongly suggest that the MS techniques can be further developed as a means for the rapid and accurate identification of bacteria.

Clinically, a bacterial count of $1 \times 10^{5}$ cells $\mathrm{mL}^{-1}$ in body fluids (other than plasma) is generally regarded as the minimum bacterial count for indication of a clinically significant infection [17]. In our sensitivity analysis in this study, we found that $1.8 \times 10^{3}$ was the minimum number of cells required for detection using MALDI-TOF MS, which is much less than $1 \times 10^{5}$. These research findings clearly indicate that microorganisms obtained from clinical samples can be subjected to MS analysis using only separation and concentration of microorganisms, without a need for further amplification by culture. Of note, further improvement of MS instrumentation, database wealth, and analytical methods can likely further increase detection sensitivity. Collectively, our findings herein reveal the great potential for using MALDI-TOF MS as a more rapid method for direct bacterial identification.

This work was supported by the National Science and Technology Major Project (Grant No. 2008ZX10207), the Innovation Method Fund of Ministry of Science and Technology of China (Grant Nos. $2008 I M 022000$ and 2009IM030300), and the National Science and Technology Support Program (Grant No. 2008BAK41B0).

1 Somtcjemko V, Iredell J R, Gilbert G L. Pathogen profiling for disease management and surveillance. Nat Rev Microbiol, 2007, 5: $464-470$

2 Widjojoatmodjo M N, Fluit A C, Verhoef J. Rapid identification of bacteria by PCR-single-strand conformation polymorphism. Clin Microbiol, 1994, 32: 3002-3007

3 Pershing D H, Tenover F C, Versalovic J, et al. Molecular microbiology: Diagnostic principles and practice, 8th ed. Washington DC: American Society for Microbiology Press, 2003

4 Anhalt J P, Fenselau C. Identification of bacteria using mass spectrometry. Anal Chem, 1975, 47: 219-225

5 Ryzhov V, Fenselau C. Characterization of the protein subset desorbed by MALDI from whole bacterial cells. Anal Chem, 2001, 73: $746-750$

6 Demirev P A, Ramirez J, Fenselau C. Tandem mass spectrometry of intact proteins for characterization of biomarkers from Bacillus cereus T spores. Anal Chem, 2001, 73: 5725-5731

7 Warscheid B, Fenselau C. A targeted proteomics approach to the rapid identification of bacterial cell mixtures by matrix-assisted laser desorption/ionization mass spectrometry. Proteomics, 2004, 4: 28772892

8 Pribil P A, Patton E, Black G, et al. Rapid characterization of Bacil- 
lus spores targeting species-unique peptides produced with an atmospheric pressure matrix-assisted laser desorption/ionization source. J Mass Spectrom, 2005, 40: 464-474

9 Sauer S, Freiwald A, Maier T, et al. Classification and identification of bacteria by mass spectrometry and computational analysis. PLoS One, 2008, 3: e2843

10 Carbonnelle E, Beretti J L, Cottyn S, et al. Rapid identification of Staphylococci isolated in clinical microbiology laboratories by matrix-assisted laser desorption ionization-time of flight mass spectrometry. J Clin Microbiol, 2007, 45: 2156-2161

11 Mellmann A, Cloud J, Maier T, et al. Evaluation of matrix-assisted laser desorption ionization-time-of-flight mass spectrometry in comparison to 16S rRNA gene sequencing for species identification of nonfermenting bacteria. J Clin Microbiol, 2008, 46: 1946-1954

12 Piseth S, Michel D, Frederique G, et al. Ongoing revolution in bacteriology: Routine identification of bacteria by Matrix-assisted laser desorption ionization time-of-flight mass spectrometry. Clin Infect
Dis, 2009, 49: 543-551

13 Torsten S. Whole-cell matrix-assisted laser desorption/ionization mass spectrometry for rapid identification of bacteriocin/lantibiotic-producing bacteria. Rapid Commun Mass Spectrom, 2008, 22: 1146-1152

14 Hoben H J, Somasegaran P. Comparison of the pour, spread and drop plate methods for enumeration of Rhizobium spp. in inoculants made from presterilized peat. Appl Environ Microbial, 1982, 44: 12461247

15 Song Y S, Talaty N, Tao W A, et al. Rapid ambient mass spectrometric profiling of intact, untreated bacteria using desorption electrospray ionization. Chem Commun, 2007, 1: 61-63

16 Lindon J C, Holmes E, Nicholson J K. Metabonomics techniques and applications to pharmaceutical research \& development. Pharm Res, 2006, 23: 1075-1088

17 Hsieh S Y, Tseng C L, Lee Y S, et al. Highly efficient classification and identification of human pathogenic bacteria by MALDI-TOF MS Mol Cell Proteomics, 2008, 7: 448-456

Open Access This article is distributed under the terms of the Creative Commons Attribution License which permits any use, distribution, and reproduction in any medium, provided the original author(s) and source are credited. 